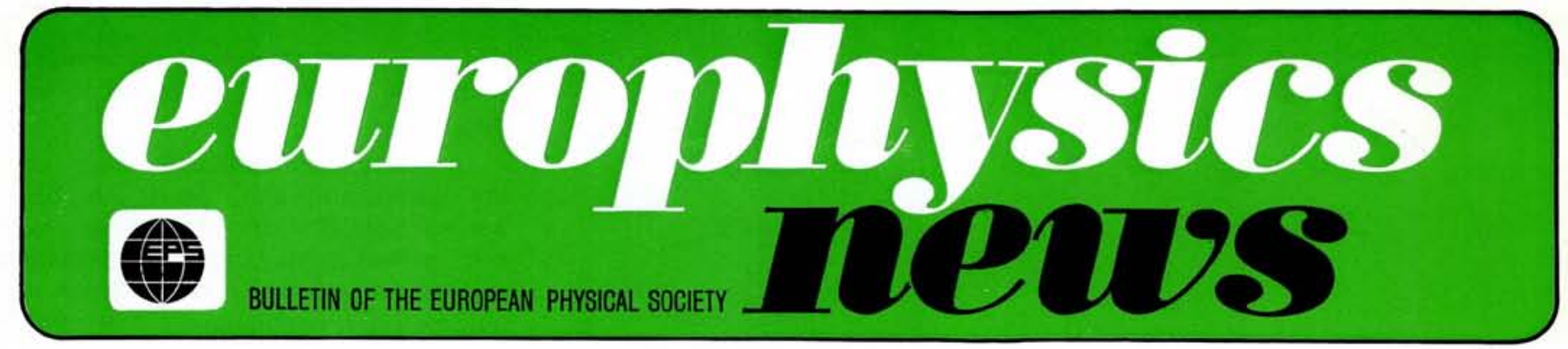

\title{
Sources of Cosmic X-rays
}

Our Galaxy is composed mostly of stars, gas and dust. The more massive stars evolve faster than less massive ones, and probably explode when their nuclear fuel is depleted. The resultant supernova ejects a large shell of matter and relativistic particles out into the surrounding interstellar gas. Shock waves propagate outward sweeping gas up and heating it to temperatures of millions of degrees, hot enough to emit X-rays.

Perhaps up to one half of all stars in our Galaxy are at least double, i.e. composed of two or more objects orbiting a common centre of mass. Such binary and triple systems display a large spectrum of separations, some containing stars actually in contact with each other, with orbital periods of a few days. If we now examine a close binary system, mass transfer from one component to the other may be observed. Stars swell up at various stages of their evolutionary cycle, and if the outer atmosphere tends to protrude above the common equipotential of the pair of stars, then this matter will be shared by the stars. If the companion star is not yet filling this equipotential surface (called the Roche surface in the case when the rotation periods of the stars are locked to their orbital periods), then the sharing is almost completely a one-way process. Such an effect obviously plays havoc with the evolutionary path of both stars, since the more massive star will evolve first, transfer mass and perhaps end up as the less massive star.

Let us now skip the intermediate and end phases of the companion that evolves first, and consider the end product. A supernova may have occurred, leaving behind a stellar

\section{A. C. Fabian, Cambridge}

A.C. Fabian is an S.R.C. Postdoctoral Research Fellow working in X-ray astronomy at the Institute of Astronomy, Cambridge, UK

fragment such as a neutron star, or the 'dead' star could have collapsed into a white dwarf, or black hole. Neutron stars are the answer to a physicist's dream, or nightmare, depending upon his outlook. One solar mass or so, compacted into a sphere of about $10 \mathrm{Km}$ radius - neutron rich matter due to an unavailability of states giving nuclear densities throughmost of the core. In a sense a misnomer, since its structure more closely resembles that of a planet rather than a star, having a solid, crystalline crust, liquid interior and perhaps a solid core. If any significant fraction of the stellar magnetic field, frozen into the matter, collapses with the imploded stellar core, then the neutron star may have surface fields of $10^{12}$ gauss. Similarly, if the original angular momentum of the star is retained by the neutron star, it will rotate every millisecond or so. Lone, highly magnetised, rotating neutron stars are detectable as pulsars. The pulsar in the Crab supernova remnant, which exploded 920 years ago (our time) emits flashes of radio, optical and $\mathrm{X}$-radiation, the exact origin of which is still a debated topic, despite intensive study.

White dwarfs are supported from further collapse by electron degeneracy pressure, having masses again similar to that of the Sun, but radii of several thousands of Kilometres. Black holes are the ultimate in collapse phenomena, being of any mass and having radii that scale linearly with mass, - about $3 \mathrm{Km}$ for one solar mass. This radius, the Schwarzschild radius in the case of a non-rotating black hole, is that below which the collapsing star will be out of contact with the rest of the Universe. In the case of a black hole formed in a close binary system, a more complicated rotating black hole is expected to form.

Now consider the situation when the second star evolves sufficiently so that mass transfer recurs (not necessarily for the second time). This time the compact component offers a deep gravitational well to any matter coming its way. If this matter were to fall directly onto a neutron star, temperatures of $10^{9} \mathrm{~K}$ or more would be realised. Of course, nothing similar would be obtained from a black hole, since the matter would apparently never strike the collapsed surface. However, conservation of angular momentum prevents mass transfer from being radial, since the objects are orbiting one another, and some form of disk will be formed around the compact object, out of the accreted material.

Mass transfer does not necessarily require overflow of equipotentials, since hot young stars have very substantial stellar winds, akin to the solar wind. A compact object bathed in such a wind from a nearby companion may form an accretion wake which could feed matter into a disk.

Viscous effects will cause a gradual transfer of angular momentum outward and consequent inward flow of

\section{Contents}

Sources of Cosmic X-rays .

Radioactive Pollution of the Environment in the Context of the Energy Problem

Society News

Meetings
1

4 7 


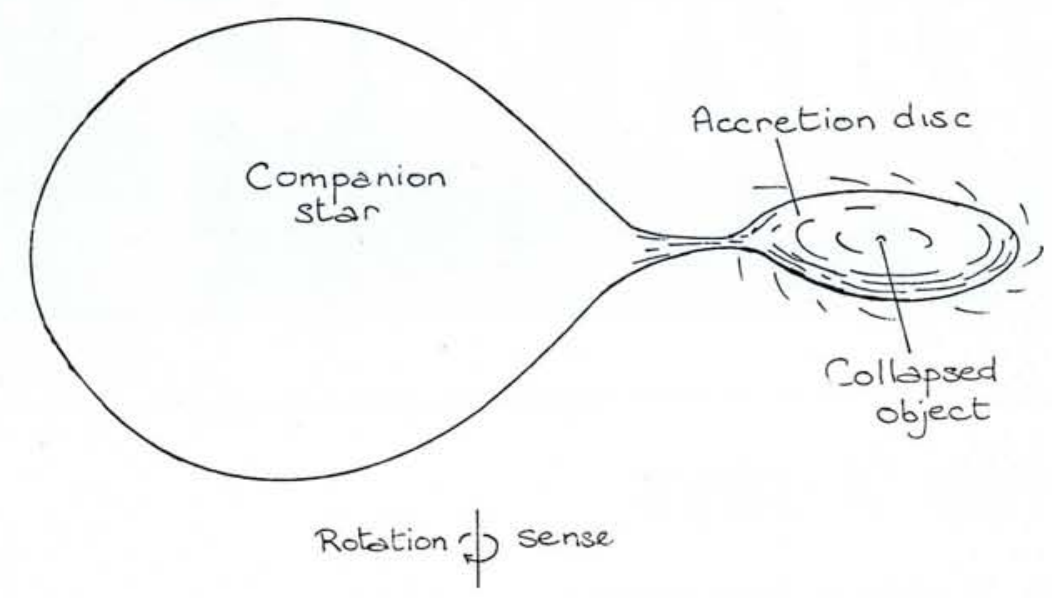

Mass transfer in a close binary system. X-rays originate either in the accretion disc close to the collapsed object, or on impact onto the surface. It the collapsed object is a magnetised neutron star, pulsed X-ray emission may be observed.

matter within the accretion disk. Shocks, turbulence, etc. will heat the inner regions of the disk to X-ray temperatures. Final accretion onto the body of the compact object will result in further release of gravitational energy as X-rays. An accretion disk surrounding a black hole will enable some gravitational energy to be extracted before the distorting effects close to the Schwarzschild radius came into play.

Cosmic X-ray astronomy, started in 1962, has revealed the presence of such phenomena within our own and nearby galaxies. Thermal $\mathrm{X}$-rays require million degree or hotter temperatures, and these occur in supernova shock waves, and if a sufficient amount of material (about $10^{-8}$ solar masses/year) is accreted by a compact object. Relativistic electrons interacting with matter, magnetic fields or photon fields can also produce X-rays, and such processes have been used to explain other events observed by X-ray astronomers.

Before 1970, observations in X-ray astronomy were almost wholly restricted to the use of rockets. Only a few minutes of observation time is afforded by the flight of a soundingrocket, but nevertheless some scores of X-ray sources were pinpointed to various degrees of accuracy. The first Small Astronomical Satellite, renamed Uhuru after launch in December 1970 was totally devoted by cosmic X-ray astronomy. Maximum collecting area was packed in by the use of proportional counters with slat collimators defining fields of view of $1 / 2^{\circ} \times 5^{\circ}$, and $5^{\circ} \times 5^{\circ}$. The satellite was spinning, and the axis of spin could be aligned with required positions, the detectors looking out normal to this axis. Total observing time on individual sources, in the course of the first year, was increased to at least a few minutes for most, and hours for others. A catalogue of almost 150 sources has been produced, indicating about 70 galactic sources which are probably of the supernova remnant of close binary, collapsed object classes.

The ability to observe the same source again and again revealed the variability of many of these galactic sources, some of which appeared to be periodic. In fact for six of these sources, the large scale variations are directly attributable to eclipses of of the X-ray source, presumably the compact object, orbiting a much larger companion star at a close distance. Such a proposal is verified by the exact agreement given by doppler shifts of pulsations of the X-ray source itself.

A typical example is Hercules $\mathrm{X}-1$. Here, a rotating magnetised neutron star is orbiting an otherwise mediocre star, with a period of 1.7 days. Mass transfer, probably by equipotential overflow, fills an accretion disk surrounding the neutron star. Magnectic fields dominate the inner disk causing funnelling of the accreted material onto the magnetic poles of the neutron star. It is here that the bulk of the observed X-ray emission originates, and due to an offset between magnetic and rotation axes, we only see one magnetic pole, and hence X-rays, for part of the 1.24 second (or 2.48 ) rotation period. The observed $\mathrm{X}$-ray emission is thus pulsed, with an observed period that changes sinusoidally due to the orbital motion of the neutron star.
In fact this enables many parameters about the dynamical situation to be deduced.

The X-rays significantly heat up the companion star, previously observed optically and known as $\mathrm{HZ}$ $\mathrm{Her}$, so that it too varies in brightness with a period of 1.7 days. What is not as yet unambiguously explained is a further period of 35 days associated with this system. The X-ray pulses are only observed for 12 out of every 35 days, whereas the light variations of $\mathrm{HZ} \mathrm{Her,} \mathrm{although} \mathrm{under-}$ going subtle changes, do not disappear completely. Perhaps some instability is associated with the mass transfer process.

Other binary X-ray sources, such as Cygnus $\mathrm{X}-3$ do not as yet have observed optical counterparts. This is probably due to the significant reddening occurring in the galactic plane due to dust. Cygnus $X-3$ is an infrared object, however, and has been detected to vary with the same 4.8 hour periodicity as the $\mathrm{X}$-ray source. Radio emission has also been detected, although its origin, and especially that of the outburst of September 1972 associated with the system, has yet to be tied in with the $\mathrm{X}$-ray emission. It could be that the $\mathrm{X}$-ray emission is not related, since other binaries emit radio waves, e.g. Algol, although no significant $X$-ray emission has yet been found from that. Cyg X-3 probably operates by virtue of a stellar wind, which is ionised by the $\mathrm{X}$-rays, and giving rise to at least some of the infrared radiation.

Cygnus $\mathrm{X}-1$, an $\mathrm{X}$-ray source with associated radio emission is identified with a massive binary system. No X-ray eclipses have as yet been published, but indirect evidence directly points to the 30 or so solar mass, HD226868, as the companion star to the X-ray source. Doppler shifts of its spectral lines, coupled with analysis of other spectral features leads to the conclusion that the compact companion is at least 10 solar masses. The only compact object of that mass is a black hole, so Cygnus $\mathrm{X}-1$ has been lauded as the first detected black hole. Such a step has not been without its critics, although it does appear that the most plausible way in which to detect a black hole is by its surrounding $\mathrm{X}$-ray emission. Further study of this interesting object may help to rule out possible alternatives such as a triple system containing a neutron star, and establish the real existence of ultimate collapse. 
It is quite probable that many more binary systems will be discovered, some of which may not show eclipses due to the inclination of their orbital planes to our line of sight. Pulses need only occur if the magnetic field is sufficiently strong so that mass flow is influenced. An observed sharp upper limit in the luminosity of binary $\mathrm{X}$-ray sources may result from radiation pressure of outgoing photons quelling the accretion process. If this upper limit can be detected in distant galaxies, and is due to the same cause, then an independent method for distance measurement will become available.

Grazing-incidence X-ray telescopes have been used on board the Orbiting Astronomical Observatory, Copernicus to map the X-ray emission from several supernova remnants in our Galaxy, as well as the location and study of binary sources. Future emissions with much larger apertures may easily resolve all the individual luminous X-ray sources in nearby Galaxies. The resolution of an X-ray telescope is more likely to be limited by photon statistics or pointing capability as opposed to aperture size.

The formation of galaxies from gas is hardly likely to be a perfectly efficient process, and it is probable that some gas will be left over between the galaxies. Limits on the density of such a gas have been made by astronomers working at various wavelenghts. These suggest that any substantial amount of matter should be hotter than a million degrees or so. X-ray emission has been detected from a number of clusters of galaxies, and the extent and spectrum of this radiation implies that hot intergalactic gas is responsible. The temperature of this gas is similar to that expected if the velocities of the gas atoms is of the same magnitude as the random velocities of individual galaxies in the cluster.

A few individual unusual galaxies have been detected, including the radio galaxy Cen $A$, the Seyferts NGC 4151 and 1275, and the quasistellar object 3 C273. The X-ray emission in these cases may plausibly originate within the galactic nuclei, by non-thermal processes. Large orbiting $\mathrm{X}$-ray telescopes in the next decade may help to define the $X$-ray emission processes, and investigate some of the most energetic phenomena yet discovered.

A diffuse background radiation exists, at least extending from photon energies of $100 \mathrm{eV}$ to $200 \mathrm{MeV}$. It is almost certainly extragalactic over the $2-100 \mathrm{KeV}$ range, and may easily

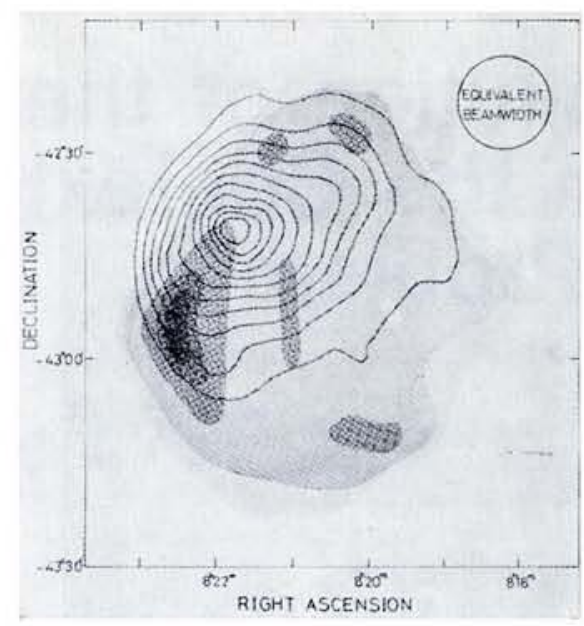

The line contours map the $\mathrm{X}$-ray emission from the supernova remnant Puppis $A$, as observed by Zarnecki et al. (1973) with $X$-ray telescopes on Copernicus. The hatched contours show the radio emission, the peaks of which do not coincide with the X-ray peak.

be of cosmological significance, resulting from radiation processes occurring in earlier phases of the Universe.

X-ray astronomy has blossomed quickly and revealed many new facets of our cosmic environment. Strong gravitational fields such as are associated with black holes, may perhaps be indirectly studied through the $\mathrm{X}$-ray emission of accreted matter. In many of the sources discussed, the X-ray emission absorbs a large fraction of the energy budget, and seriously affects the evolution and lifetime. European astronomers have not been slow to realise the importance of X-ray astronomy, with many groups participating both observationally in sounding-rocket and balloon studies, and theoretically. British experience with the grazingincidence telescopes and X-ray detectors on Copernicus will be followed by the satellites UK- 5 and -6 ; the Dutch will have their X-ray satellite ANS in orbit soon, and there are joint European ventures in EXOSAT and similar projects. X-ray astronomy may prove expensive but cannot be ignored.

\section{REFERENCES}

1) Bradt, H., and Giacconi, R. (Editors), 'Non Solar X-and $\gamma$-ray Astronomy, IAU Symposium 55, Dordrecht (1973)

2) Pringle, J.E., and Rees, M.J., 'Accretion Disc Models for Compact X-ray Sources', Astronomy and Astrophysics, vol. 21, p. 1. (1972).

3) Giacconi, R., Gursky, H., Kellogg, E., Levinson, R., Schreier, E., and Tananbaum, H., 'Further X-ray Observations of Hercules X-1 from Uhuru', Astrophysical Journal, vol. 184. p. 227 (1973)

4) Zarnecki, J.C., Culhane, J.L., Fabian, A.C. Rapley, C.G., Silk, R., Parkinson, J.H., and Pounds K.A. 'Low Energy X-ray Map of the Puppis A Supernova Remnant', Nature Physical Science, vol. 243 , p. 4. (1973),
New Books from North-Holland

\section{Cosmic Rays \\ Variations and Space Explorations}

by L.I. DORMAN

Izmiran, Moscow, U.S.S.R.

Translated from the Russian by H.A. PelsKluyver

English translation edited by H.C. van de Hulst

\section{4. about 668 pages}

Dfl. 215.00 (about US $\$ 78.20$ )

ISBN 0-7204-0256-5

Since the appearance of the first Russian edition of this book in 1963, our knowledge of cosmic rays has been greatly increased by (1) the use of satellites and cosmic and in inter neighbourhood of the Earth abservation of the virect rays, magnetic fields and solar cosmic streams, and (2) by the installation plasma a hundred laboratories for measuring over mic-ray variations in many during the International Year of the Sun (1964-5) and the Active Sun (1968-70).

The second Russian edition (of which this is a translation) was completely overhauled to incorporate the wealth of new material and to reject any obselete information.

CONTENTS: Variations of Cosmic Rays as a Means for Investigating the Cosmos. Experimental Methods for Investigating Cosmic-Ray Variations. Meteorological Cosmic-Ray Effects (Variations of Class I). The Method of Coupling Coefficients. Geomagnetic Separation of Cosmic Rays. Cosmic-Ray Variations of Geomagnetic Origin. Problem to Determine Extra-terres trial Variations.

\section{Local Currents and their Applications}

Proceedings of the Conference held at Princeton, October 8-10, 1971

edited by A.S. WIGHTMAN, Princeton University, and D.H. SHARP, University of Pennsylvania.

\section{4. about 160 pages.}

Difl. $\mathbf{3 0 . 0 0}$ (about US $\$ \mathbf{1 0 . 9 0}$ )

ISBN 0-7204-0276-X

CONTENTS: Ideas about the role of currents in particle physics (R. Dashen), What constructive field theory says about currents (A. Jaffe and $O$. McBryan), Current commutators on the light cone (R.A. Brandt). What we have learned about representing local non-relativistic current algebras (D.H. Sharp). What we have learned about local relativistic current algebras (G.A. Goldin). Matrix element of local operators (B. Andersson). A theory of electromagnetic and weak interactions (B.W. Lee). Anomalies in Ward identities and current commutation relations (S.L. Adler).

\section{NORTH HOLLAND}

P.O. Box 211, Amsterdam, The Netherlands

Distributed in the United States and Canada by American Elsevier Publishing Vanderbilt Avenue, New York, N.Y., 10017. 